\title{
Some possible station layouts and characteristics of evacuated tube transportation
}

\author{
Yaoping $\mathrm{ZHANG}^{1 *}$, Benlin $\mathrm{LIU}^{1}$, Yong ZHAO \\ 1. Institute of Vacuum Tube Transportation, Xijing University, Xi'an 710123, China \\ 2. Superconductivity and New Energy Center (SNEC), Southwest Jiaotong University, Chengdu 610031, China
}

\begin{abstract}
Since Maglev vehicles will run in a closed vacuum tube, the layout of the terminal stations of evacuated tube transportation (ETT) will differ from the traditional railway stations. This paper deals with some possible station layouts of ETT, e.g., a station with an airlock, a station without an airlock, above ground and underground stations, and stations with either level arrayed or rotation platforms. Then different station layouts are compared, and characteristics of each are analyzed. Finally, a more secure mode for ETT station layouts is suggested, which can be the basis for future ETT station layout and designs.
\end{abstract}

Key words: station layout; terminal; vacuum; evacuated tube transportation; airlock

(C) 2011 JMT. All rights reserved.

\section{Introduction}

$\mathrm{E}$ vacuated tube transportation (ETT), compared with other traffic modes is low in energy consumption, relatively safer, not associated with air pollution, produces little noise and is much faster. It will be an ideal traffic mode in the future. Because Maglev vehicles will run in a closed vacuum tube, the station's terminal layout of ETT will differ from the traditional railway stations. There are several possible ETT station modes that can be considered, such as stations with an airlock [1-3], stations without an airlock [4], above ground or underground stations, and stations with either level arrayed platforms and a rotation platform. In fact, Pro Swissmetro in Switzerland has conceived of ETT station layouts underground. Et3.com Inc. in the United States has conceived of ETT station layouts on the ground, but they did not conduct detailed comparisons of different ETT station layouts [5-6].

This paper introduces different station layouts, and analyzes their characteristics.

In this paper, two concepts, terminal station and midway station are employed. Terminal station means the station is built where the track/tube reaches an end, and the vehicle will not pass it, but not certainly means

Received Dec. 7, 2010; revision accepted Aug. 22, 2011

${ }^{*}$ Corresponding author. Tel.: +86-29-85628051

E-mail: tubetrans@hotmail.com (Y.P.ZHANG)

(C) 2011 JMT. All rights reserved

doi: 10.3969/j.issn.2095-087X.2011.03.006 the ETT line end. Midway station may consist of two or more terminal stations.

\section{ETT station with an airlock}

In order to implement isolation between the vacuum environment inside the tube and the atmospheric environment outside the tube, an airlock is necessary. With an airlock cabin as shown in Fig. 1, ETT vehicles can go into the vacuum tube or exit the vacuum tube through an isolation gate [1-3], while air will not flow into the vacuum tube, avoiding damage to the vacuum environment.

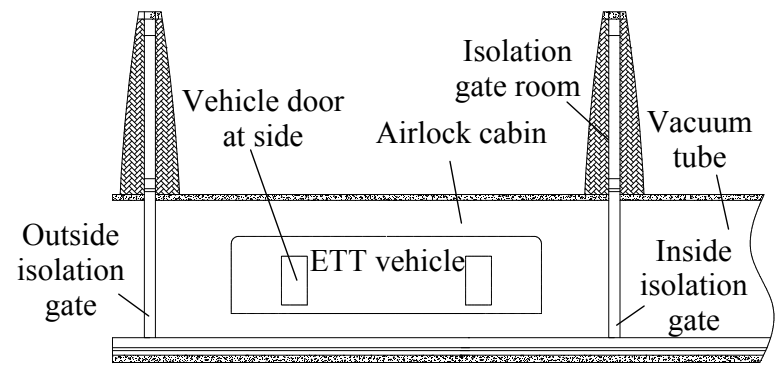

Fig. 1 Airlock of ETT

Generally, an ETT station's terminal airlock includes a cabin and two isolation gates (an inside gate and an outside gate). The inside gate is adjacent to the main vacuum tube, and the outside gate is adjacent to open space, i.e. station area. In this case, there are three modes to organize passengers to get on/off the vehicle. (1) As shown in Fig. 2(a), the vehicle goes out of the 
airlock cabin through the outside isolation gate so that passengers can get on/off the vehicle in the station area. (2) As shown in Fig. 2(b), the vehicle always stays in the airlock cabin, but the outside isolation gate is placed at the end position of the airlock and passengers get on/off the vehicle through the end door of the vehicle. (3) As shown in Fig. 2(c), the vehicle always stays in the airlock cabin, but the outside isolation gate is placed on the two sides of vehicle and passengers get on/off the vehicle through the side doors.

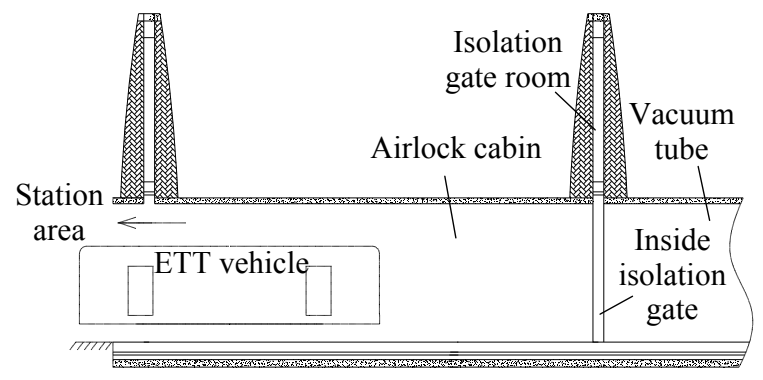

(a) ETT vehicle goes out of airlock to allow getting on/off

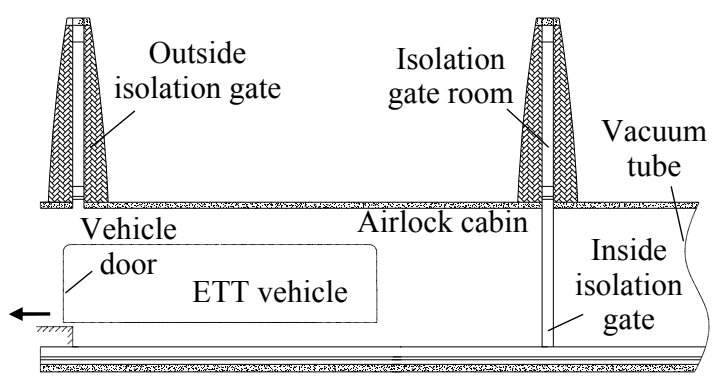

(b) Passengers get on/off at the end door of ETT vehicle

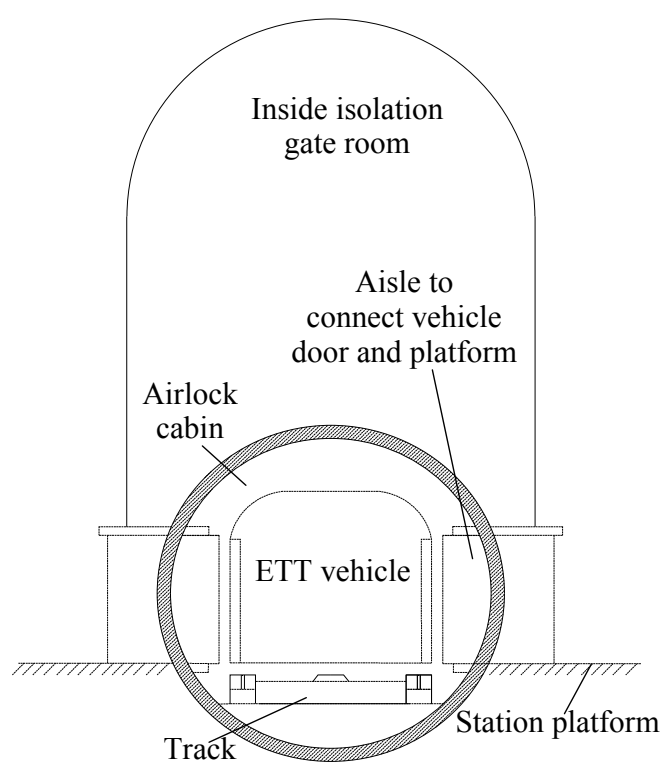

(c) Passengers get on/off at the side doors of ETT vehicle

Fig. 2 Some different modes for passengers' getting on/off the ETT vehicle

\section{ETT station without airlock}

It is possible to build an ETT terminal station or midway station without airlock. In this case, the vehicle always stays in vacuum environment and passengers get on/off the vehicle through the aisle that connects the vehicle door and platform.

As for the terminal station without an airlock, there are three possible specific modes of the station layout. (1) As shown in Fig. 3(a), the vehicle door will be at the end of the vehicle and passengers get on/off the vehicle through the aisle, which has a function to isolate air when closed; (2) as shown in Fig. 3(b), the vehicle door will be at one side; and, (3) as shown in Fig. 3(c), the vehicle doors will be on the two sides of the vehicle [4].

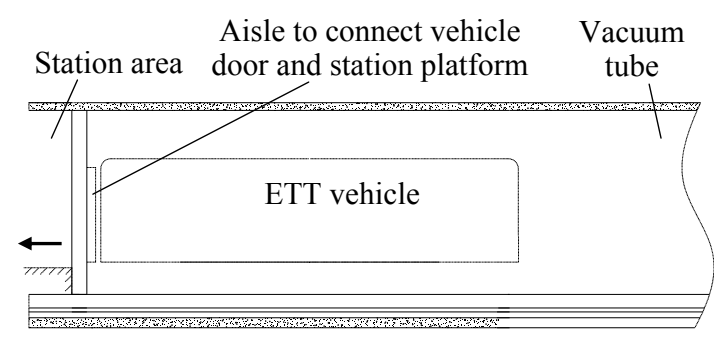

(a) Passengers get on/off at the end door of ETT vehicle

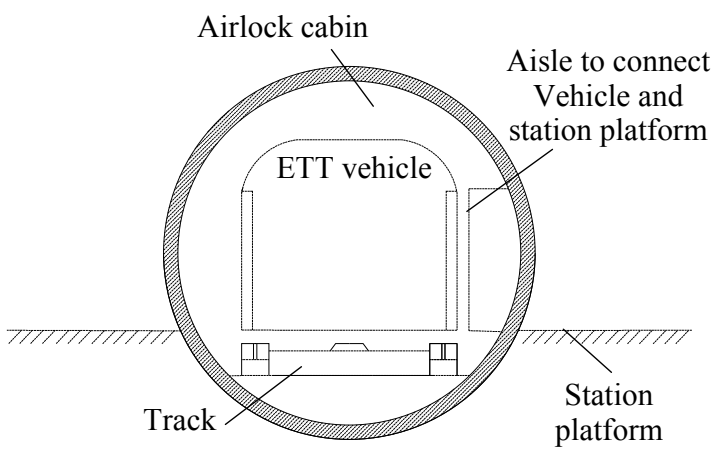

(b) Passengers get on/off at one side door of ETT vehicle

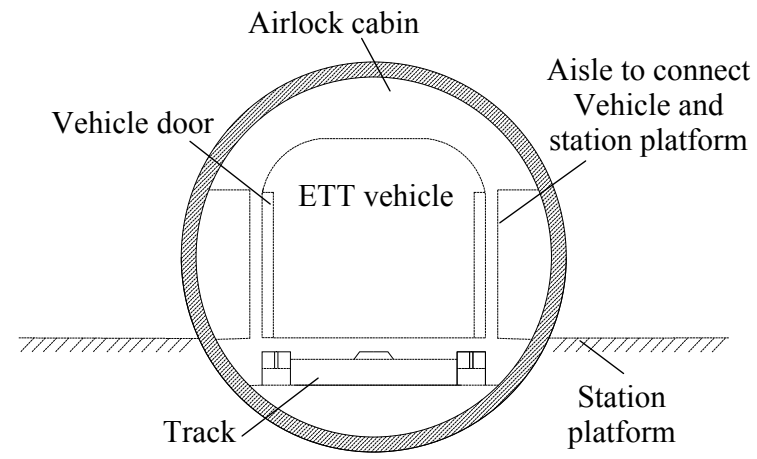

(c) Passengers get on/off at doors at two sides of ETT vehicle

Fig. 3 Terminal Station layout without airlock 
The mode with the vehicle gate at only one side of the vehicle faces a problem. The tremendous air pressure would push the vehicle forward. Therefore, a Chinese patent has given a station layout with openable gates at two sides of the vehicle as well as the tube, so that the air pressure pressed on the vehicle can be counteracted [4]. As for the mode with an end door, the air pressure on the vehicle will be less than in the mode with the door at only one side, because the transect of the vehicle is generally less than the vertical section of the vehicle.

As for the midway station without airlock, there are two possible kinds of the station layouts as shown in Fig. 4(a) and Fig. 4(b).

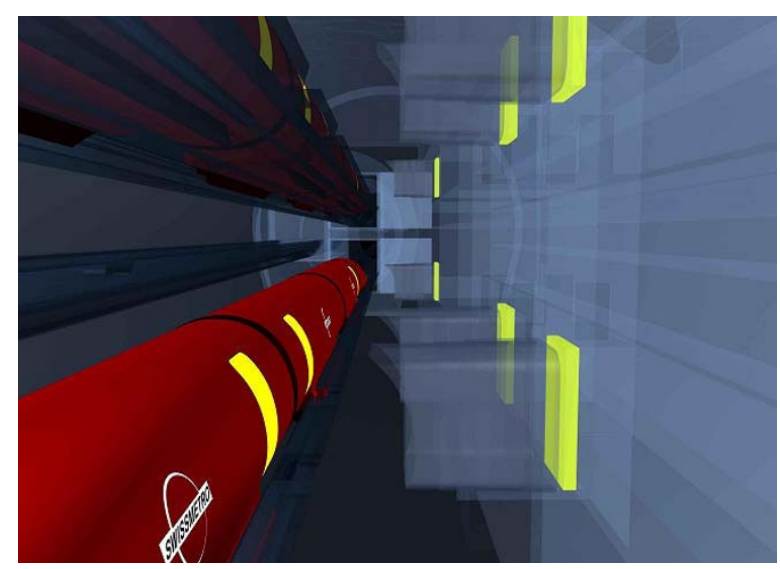

(a) Midway station layout with door and aisle at only one side of the vehicle

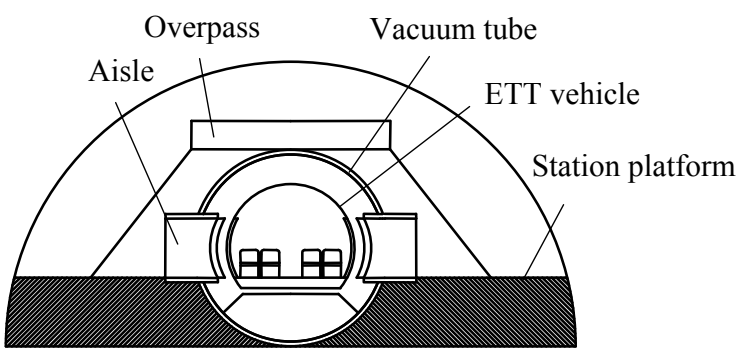

Transection cutaway view

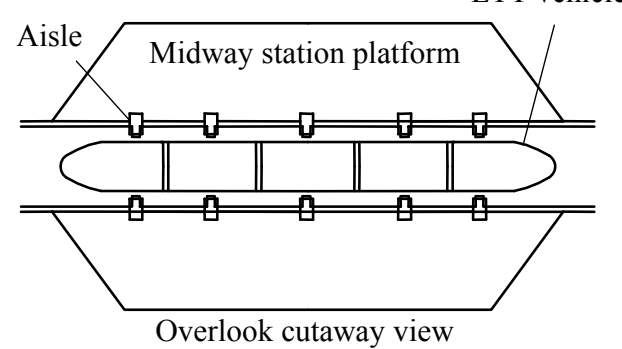

(b) Midway station layout with doors and aisles at two sides of the vehicle

Fig. 4 Midway station layout without an airlock

\section{ETT station on the ground}

Like the majority of the railway stations, the ETT station can be created on the ground. Both the terminal station and the midway station could be built on the ground so long as the ETT line passes on the ground or ETT line adjacent to ETT station region is on the ground. In fact, there are two kinds of station modes on the ground, i.e., being paved on the ground and overhead, as shown in Fig. 5 [6].



Fig. 5 Station layout on ground (Picture from http://www. et3.com)

The construction cost to build an ETT station on the ground would be less than an ETT station under ground. However, an ETT station on the ground would occupy more land.

\section{ETT station under the ground}

If the ETT line is always underground, then the ETT station (including terminal station and midway station) will certainly be underground. In this case, passengers will get on/off the vehicles by elevator (as shown in Fig. 6), or walk to the underground platform by stairs, as in a subway.

The construction cost to build an ETT station under the ground would be more than an ETT station on the ground, but occupy less land.

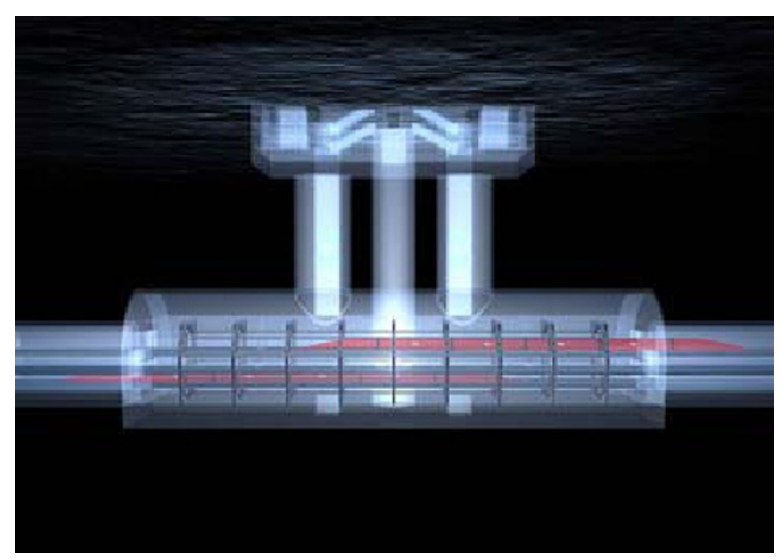

Fig. 6 Station layout under ground (picture from http: //www.swissmetro.com) 


\section{ETT station with level arrayed platforms}

Like the current traditional railway station platform, the ETT station also can be built by level layout, i.e., all tracks or station tubes will be placed on one horizontal plane (as shown in Fig. 7).



Fig. 7 Midway and terminal station layout with level arrayed platforms

Whether it is an ETT station on the ground or under the ground, the level arrayed platform will be feasible. For a midway ETT station, overhead bridge or underway aisle is necessary so that passengers go to different platforms.

\section{ETT station with tubes on a different horizontal plane}

Different from the traditional railway, it is possible to place tubes on different horizontal plane. When the ETT line is created in the multi-layered-structure mode, correspondingly, a multi-layered-structure station (terminal station or midway station) is built. As shown in Fig. 5 and Fig. 8, a multi-layered-structure station would save

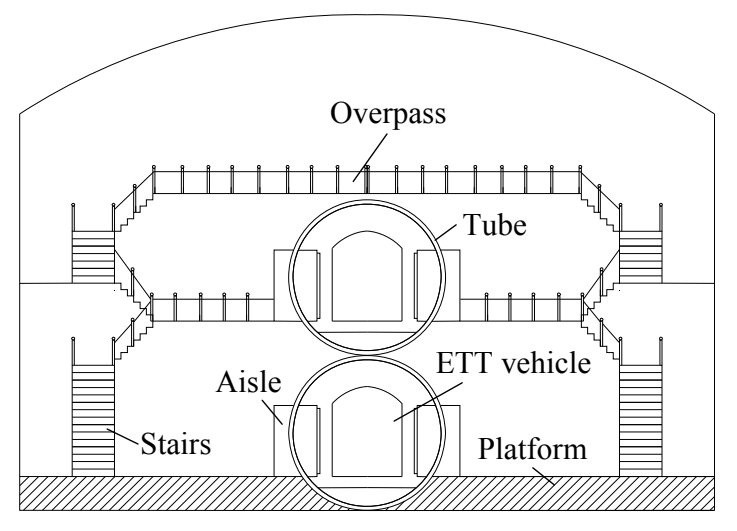

(a) Double-layered-structure station

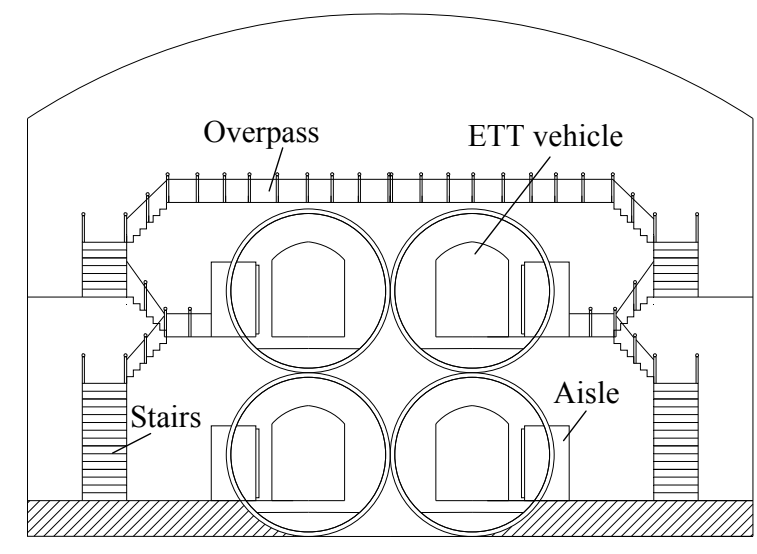

(b) Multi-tube double-layered structure station

Fig. 8 Multi-layered-structure ETT station

land use. The ETT station layout could be either underground or on the ground (including overhead).

In fact, for a cross ETT station where passengers need to transfer from one direction to another direction, a multi-layered-structure ETT station is necessary.

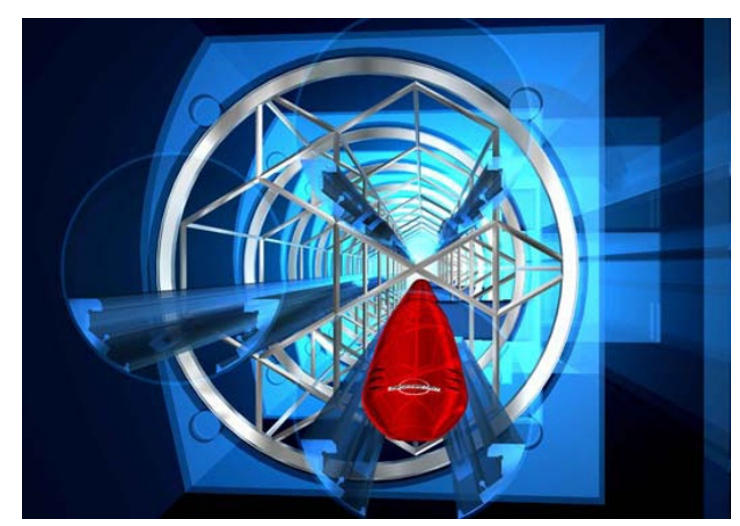

(a) Midway station without an airlock cabin (Picture from http://www.swissmetro.com)

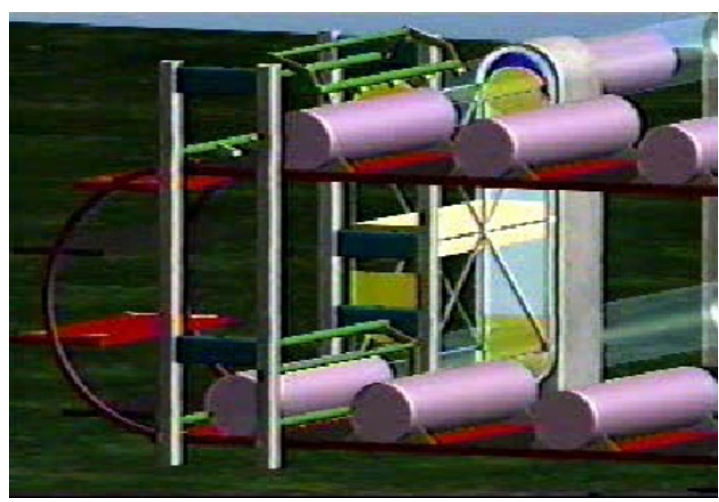

(b) Terminal station with an airlock cabin (Picture from http://www.et3.com)

Fig. 9 Station layout with rotation platform 


\section{ETT station layout with rotation platform}

In order to reduce the space occupied by the station and increase the speed with which the ETT vehicles depart or arrive, an ETT station with a rotation platform is a consideration. For the station without an airlock, the rotation platform could be planned as shown in Fig. 9(a) [5], where passengers get on/off the vehicle through the aisle which can extend to the side door of ETT vehicle. For the station with an airlock cabin, the rotation platform could be planned as shown in Fig. 9(b), and passengers get on/off the vehicle when it is outside the ETT airlock cabin.

\section{Conclusions}

All the above-mentioned ETT station layouts are feasible. The selection of the station mode will depend on the specific construction when the ETT line is built. However, some modes have apparent shortcomings, e.g., for the station layout without an airlock cabin, the station mode with the door at only one side of the vehicle is not adequately safe. Therefore, the station mode (without an airlock cabin) with doors and aisles at two sides of the vehicle is recommended. As for the terminal station layout, regardless of whether there is an airlock cabin or not, the station mode for exit and a vehicle door at the end is advisable.

\section{Acknowledgments}

This work was supported by the National Natural Science Foundation of China (No.50678152), and the Scientific Plan Fund of Shaanxi Province (No.2009K09-24).

\section{References}

[1] Y.P. Zhang, B.L. Liu, S.S. Li, et al., Levelly moveable vacuum isolation gate for evacuated tube transportation, China patent, No.2009103061881, Aug. 27, 2009.

[2] Y.P. Zhang, Y. Zhao, B.L. Liu, et al., vertically moveable vacuum isolation gate for evacuated tube transportation, China patent, No. 200910219090.2, 2009.

[3] Y.P. Zhang, B.L. Liu, S.S. Li,et al., oppositely moveable vacuum isolation gate with two doors for evacuated tube transportation, China patent, No.200910219090.2, Nov. 20, 2009.

[4] Y.P. Zhang, B.L. Liu, T.Q. Ding, et al., layout of the vehicle and the station with doors which are openable at two sides for evacuated tube transportation, China patent, No.201010176218.4, 2010.

[5] Pro Swissmetro. Swissmetro pictures. http://www. swissmetro.ch, Pro Swissmetro, Switzerland, 2011.

[6] Et3.com Inc. ETT Gallery. http://www.et3.com, Et3.com Inc., Florida, U.S., July 10, 2010.

(Editor: Dongju CHEN) 